ISSN 1392-3196 / e-ISSN 2335-8947

Zemdirbyste-Agriculture, vol. 100, No. 4 (2013), p. 441-446

DOI $10.13080 /$ z-a.2013.100.056

\title{
Baltic fruit rootstock studies: evaluation of apple (Malus domestica Borkh.) new rootstocks
}

\author{
Darius KVIKLYS ${ }^{1}$, Nomeda KVIKLIENE ${ }^{1}$, Pawel BIELICKI ${ }^{2}$, Andris BITE ${ }^{3}$, Janis LEPSIS ${ }^{3}$, \\ Toivo UNIVER ${ }^{4}$, Neeme UNIVER ${ }^{4}$, Nobertas USELIS ${ }^{1}$, Juozas LANAUSKAS ${ }^{1}$ \\ ${ }^{1}$ Institute of Horticulture, Lithuanian Research Centre for Agriculture and Forestry \\ Kauno 30, Babtai, Kaunas distr., Lithuania \\ E-mail: d.kviklys@1sdi.lt \\ ${ }^{2}$ Research Institute of Horticulture \\ Konstytucji 3 Maja 1/3, Skierniewice, Poland \\ ${ }^{3}$ Pure Horticultural Research Centre \\ Pure, Tukumas distr., Latvia \\ ${ }^{4}$ Polli Horticultural Research Centre, Institute of Agricultural and Environmental Sciences, \\ Estonian University of Life Sciences \\ Polli, Karksi-Nuia, Viljandi county, Estonia
}

\begin{abstract}
Within the framework of the project "Baltic fruit rootstock studies", apple rootstocks M.26, M.9, P 22, P 59, P 61, P 62, P 66, P 67, B.396, PB.4 and Pure 1 were tested with the cv. 'Ligol' over the years 2005-2010 in Estonia, Latvia, Lithuania and Poland. The vigour of trees on PB.4, P 61, P 59, P 22 and Pure 1 rootstocks was lower than that on M.9; on P 62, P 66, P 67 and B.396 it was similar to that on M.9, and more vigorous on M.26. Apple trees on the most dwarfing P 22, P 59, P 61 and PB.4 rootstocks together with Pure 1 and P 67 had significantly higher cumulative yield efficiency on average over all locations. Nevertheless rootstock effect on the cumulative yield efficiency was modified by the effect of trial location. Rootstock effect on fruit weight was relatively low. In average, P 62 developed the biggest fruit weight, whilst the lowest fruit weight was recorded on P 59. Results of rootstock effect on fruit colouring varied among trial locations. Due to desirable growth vigour, high yield and yield efficiency, rootstocks P 67 and B.396 are a good replacement of M.9 in the areas where high winterhardiness of rootstocks is required.
\end{abstract}

Key words: fruit quality, growth, yield, yield efficiency.

\section{Introduction}

A search for the ideal rootstock for apples is continuing. Despite a wide range of growth classes, dwarfing rootstocks of M.9 growth vigour are most important for high density apple orchards. Knowing some disadvantages of M.9, especially its lower winterhardiness, other dwarf and super-dwarf rootstocks are being tested in different apple growing countries. Nowadays additional resistances against, e.g., collar rot and fireblight, and other biotic and abiotic stresses are requested and integrated in rootstock breeding programs (Hrotkó, 2007; Fazio et al., 2011).

A new apple rootstock breeding programme was started in 1975 at the Research Institute of Horticulture, Skierniewice, Poland in order to obtain hybrids resistant to collar rot diseases. The most promising dwarfing clones P 61, P 62, P 66 and P 67 were released (Czynczyk, Jakubowski, 2007). Trials with new rootstocks were established in Poland and very first testing results have been available until now (Bielicki et al., 2007; Zurawicz et al., 2011).
A super-dwarfing rootstock PB.4 was bred in Brest Horticultural Research Station, Belarus and registered in 1999 as a winterhardy rootstock. Only few trials with this rootstock have been carried out until now (Sadowski et al., 2000; Samus et al., 2006; Jadczuk et al., 2007; Piestrzeniewicz et al., 2009), therefore recommendations for its use cannot be provided yet. A dwarfing rootstock Pure 1 bred in Pure Horticultural Research Station, Latvia showed promising results in previous Baltic fruit rootstock studies (Kviklys et al., 2012).

Rootstock researchers from different countries stress the importance of multi-location trials. The programme "Baltic fruit rootstock studies" united horticulture institutes and research centres near the Baltic Sea and is open for new initiatives.

The objective of the research was to assess the effect of new or not widespread promising rootstocks on growth and productivity of apple trees within the framework of the international programme "Baltic fruit rootstock studies". 


\section{Materials and methods}

The trials were carried out at the Research Institute of Horticulture located at Skierniewice, Poland $\left(51^{\circ} 58^{\prime} \mathrm{N}, 20^{\circ} 09^{\prime} \mathrm{E}\right)$, Institute of Horticulture, Lithuanian Research Centre for Agriculture and Forestry in Babtai, Lithuania $\left(55^{\circ} 60^{\prime} \mathrm{N}, 23^{\circ} 48^{\prime} \mathrm{E}\right)$, Pure Horticultural Research Centre in Latvia $\left(57^{\circ} 02^{\prime} \mathrm{N}, 22^{\circ} 52^{\prime} \mathrm{E}\right)$ and at Polli Horticultural Research Centre, Institute of Agricultural and Environmental Sciences of the Estonian University of Life Sciences in Estonia $\left(58^{\circ} 67^{\prime} \mathrm{N}, 25^{\circ} 33^{\prime}\right.$ E) in the years 2005-2010.

Eleven vegetatively propagated apple rootstocks M.26, M.9, P 22, P 59, P 61, P 62, P 66, P 67, B.396, PB.4 and Pure 1 were tested with the cv. 'Ligol'. Planting material was produced in Arno nursery in Poland. The orchards were planted in the spring of 2005 under a uniform scheme. Planting distance was $4 \times 1.5 \mathrm{~m}$. Trees were trained as slender spindle. Pest and disease management was carried out according to the rules of integrated plant protection. The trials were arranged in a randomized block design, with four replicates and three trees per plot. Tree growth was evaluated by measuring the trunk diameter, $30 \mathrm{~cm}$ above soil surface, converted to trunk cross sectional area (TCSA) in $\mathrm{cm}^{2}$. Fruit yield $(\mathrm{kg})$ and mean fruit weight $(\mathrm{g})$ from each tree were recorded and for data analysis the averages per tree of a replicated plot were calculated. Cumulative yield efficiency was calculated as a ratio of yield per tree to TCSA and expressed in $\mathrm{kg} \mathrm{cm}^{-2}$. The relative tree size, yield, yield efficiency and fruit weight were calculated as percentages of the respective parameters of the trees on M.9 rootstock - considered as standard (100\%). Fruit blush (surface red colour) was estimated on 50 fruits per tree by visual evaluation and expressed as percentage of skin covered with red blush.

Climate conditions and soil properties differed between trial locations. In Poland: soil - deep sandyloam, $\mathrm{pH}-6.7$, humus $-1.4 \%, \mathrm{P}_{2} \mathrm{O}_{5}-195 \mathrm{mg} \mathrm{kg}{ }^{-1}, \mathrm{~K}_{2} \mathrm{O}$ - $190 \mathrm{mg} \mathrm{kg}^{-1}$, average annual precipitation $-550 \mathrm{~mm}$, average sum of active temperatures $-2550^{\circ}$. In Lithuania: soil - clay loam, $\mathrm{pH}-7.3$, humus $-2.8 \%, \mathrm{P}_{2} \mathrm{O}_{5}-255$ $\mathrm{mg} \mathrm{kg}{ }^{-1}, \mathrm{~K}_{2} \mathrm{O}-230 \mathrm{mg} \mathrm{kg}^{-1}$, average annual precipitation $-630 \mathrm{~mm}$, average sum of active temperatures $\left(>10^{\circ} \mathrm{C}\right)$ $-2300^{\circ}$. In Latvia: soil - loamy sand, $\mathrm{pH}-6.4$, humus $-1.8 \%, \mathrm{P}_{2} \mathrm{O}_{5}-290 \mathrm{mg} \mathrm{kg}^{-1}, \mathrm{~K}_{2} \mathrm{O}-180 \mathrm{mg} \mathrm{kg}^{-1}$, average of year precipitations $-640 \mathrm{~mm}$, average sum of active temperatures $-2100^{\circ}$. In Estonia: soil - clay loam, $\mathrm{pH}$ - 5.3, humus - 2.0\%, $\mathrm{P}_{2} \mathrm{O}_{5}-130 \mathrm{mg} \mathrm{kg}{ }^{-1}, \mathrm{~K}_{2} \mathrm{O}-223$ $\mathrm{mg} \mathrm{kg}{ }^{-1}$, average annual precipitation $-455 \mathrm{~mm}$, average sum of active temperatures $-1930^{\circ}$. Apple yield and mean fruit weight was not evaluated in Latvia in 2007 due to spring frost damage. High yield losses caused by the same reason were recorded in Poland in 2009 and Estonia in 2010.

The data on the main traits were subjected to the analysis of variance. Significance of differences between treatment (rootstock) means was evaluated using Duncan's multiple range test at $P<0.05$. Because of the inherent differences in variance among locations, rootstock effects were analysed for each location separately.

\section{Results}

Tree growth. On average, most vigorous tree growth was recorded in Lithuania, weaker in Poland, Latvia and Estonia (Table 1). The average growth of all rootstocks in Lithuania exceeded the average of other trial locations by $25-40 \%$. In spite of that, relative differences among most of the rootstocks within location were similar: the strongest 'Ligol' apple tree growth was on M.26 in all locations, the weakest - on PB.4, P 59, P 61 and $\mathrm{P} 22$. The size of the trees on M.26 rootstock did not differ significantly from that on M.9 in Poland, whereas in Lithuania and Estonia it was $43 \%$ and $80 \%$ higher, respectively. Some interactions occurred with P 62 too. Its growth was similar to M.9 in Lithuania, but significantly stronger than M.9 in Estonia and significantly lower in Poland and Latvia.

Table 1. Rootstock effect on apple tree size $\left(\mathrm{cm}^{2}\right)$ in 2010, expressed as the tree cross sectional area (TCSA)

\begin{tabular}{ccccccccccc}
\hline Rootstock & Poland & $\begin{array}{c}\text { Relative } \\
\text { size } \%{ }^{1}\end{array}$ & Lithuania & $\begin{array}{c}\text { Relative } \\
\text { size } \%\end{array}$ & Latvia & $\begin{array}{c}\text { Relative } \\
\text { size } \%\end{array}$ & Estonia & $\begin{array}{c}\text { Relative } \\
\text { size \% }\end{array}$ & $\begin{array}{c}\text { Average } \\
\text { over four } \\
\text { locations }\end{array}$ & $\begin{array}{c}\text { Relative } \\
\text { size \% }\end{array}$ \\
\hline M.9 & $18.5 \mathrm{ab}^{2}$ & 100 & $24.6 \mathrm{bc}$ & 100 & $19.6 \mathrm{~b}$ & 100 & $15.2 \mathrm{bc}$ & 100 & $19.5 \mathrm{~b}$ & 100 \\
M.26 & $20.0 \mathrm{a}$ & 108 & $35.2 \mathrm{a}$ & 143 & $24.8 \mathrm{a}$ & 126 & $27.3 \mathrm{a}$ & 180 & $26.8 \mathrm{a}$ & 138 \\
P 22 & $9.1 \mathrm{e}$ & 49 & $15.2 \mathrm{ef}$ & 62 & $15.4 \mathrm{~cd}$ & 79 & $9.1 \mathrm{ef}$ & 60 & $12.2 \mathrm{de}$ & 63 \\
P 59 & $5.1 \mathrm{f}$ & 28 & $10.7 \mathrm{~g}$ & 44 & $14.2 \mathrm{~d}$ & 72 & $9.6 \mathrm{ef}$ & 63 & $9.9 \mathrm{ef}$ & 51 \\
P 61 & $7.9 \mathrm{ef}$ & 43 & $13.8 \mathrm{efg}$ & 56 & $9.0 \mathrm{e}$ & 46 & $5.3 \mathrm{~g}$ & 35 & $9.0 \mathrm{ef}$ & 46 \\
P 62 & $11.0 \mathrm{de}$ & 59 & $26.4 \mathrm{~b}$ & 107 & $16.0 \mathrm{~cd}$ & 81 & $18.1 \mathrm{~b}$ & 119 & $17.9 \mathrm{bc}$ & 92 \\
P 66 & $14.5 \mathrm{c}$ & 78 & $21.2 \mathrm{~cd}$ & 86 & $14.3 \mathrm{~d}$ & 73 & $11.3 \mathrm{de}$ & 75 & $15.3 \mathrm{bcd}$ & 79 \\
P 67 & $14.8 \mathrm{c}$ & 80 & $24.6 \mathrm{bc}$ & 100 & $18.3 \mathrm{bc}$ & 93 & $15.9 \mathrm{bc}$ & 105 & $18.4 \mathrm{bc}$ & 94 \\
PB.4 & $6.1 \mathrm{ef}$ & 33 & $10.7 \mathrm{~g}$ & 44 & $6.9 \mathrm{e}$ & 35 & $7.5 \mathrm{fg}$ & 50 & $7.8 \mathrm{f}$ & 40 \\
B.396 & $15.7 \mathrm{bc}$ & 85 & $26.4 \mathrm{~b}$ & 107 & $19.2 \mathrm{~b}$ & 98 & $13.8 \mathrm{~cd}$ & 91 & $18.8 \mathrm{c}$ & 96 \\
Pure 1 & $13.0 \mathrm{~cd}$ & 70 & $17.3 \mathrm{de}$ & 70 & $13.8 \mathrm{~d}$ & 70 & $13.8 \mathrm{~cd}$ & 91 & $14.5 \mathrm{~cd}$ & 74 \\
Mean & 12.3 & & 20.6 & & 15.6 & & 13.4 & & 15.5 & \\
\hline
\end{tabular}

Note. ${ }^{1}$ - the TCSA of trees on M.9 rootstock was considered as $100 \% ;{ }^{2}$ - means followed by the same letter in each column are not significantly different at $P \leq 0.05$ by Duncan's multiple range test. 
On average, vigour of the trees on PB.4, P 61, P 59, P 22 and Pure 1 rootstocks was lower than on M.9; on P 62, P 66, P 67 and B.396 the vigour was the same as on M.9, and trees on M.26 were more vigorous than on M.9.

Yield and yield efficiency. The highest cumulative yield (2006-2010) per tree was obtained in Lithuania followed by Estonia, Poland and Latvia (Table 2). Yields per tree tended to be closely related to tree size; therefore rootstocks inducing the largest trees induced also the highest yield. Apple trees on M.26 rootstock produced significantly higher yield in Poland and Estonia, but not in Lithuania where no rootstock topped B.396 and M.9.

On average, the five rootstocks that exhibited the highest growth vigour (M.26, M.9, P 67, P 62 and B.396) had the highest cumulative yields, too. Regarding locations, yield from trees on rootstock P 66 and Pure 1 in Poland and on rootstock P 66 in Estonia did not differ significantly from mentioned rootstocks above. The lowest cumulative yield in all locations was on PB.4 followed by trees on P 61 and P 59 rootstocks. All these

Table 2. Rootstock effect on cumulative yield $\left(\mathrm{kg}\right.$ tree $\left.{ }^{-1}\right), 2006-2010$

\begin{tabular}{|c|c|c|c|c|c|c|c|c|c|c|}
\hline Rootstock & Poland & $\begin{array}{l}\text { Relative } \\
\text { yield } \%{ }^{1}\end{array}$ & Lithuania & $\begin{array}{l}\text { Relative } \\
\text { yield \% }\end{array}$ & Latvia & $\begin{array}{l}\text { Relative } \\
\text { yield \% }\end{array}$ & Estonia & $\begin{array}{l}\text { Relative } \\
\text { yield \% }\end{array}$ & $\begin{array}{l}\text { Average } \\
\text { over four } \\
\text { locations }\end{array}$ & $\begin{array}{l}\text { Relative } \\
\text { yield \% }\end{array}$ \\
\hline M.9 & $28.3 b^{2}$ & 100 & $62.8 \mathrm{a}$ & 100 & $8.29 \mathrm{~b}$ & 100 & $33.0 \mathrm{~b}$ & 100 & $33.1 \mathrm{ab}$ & 100 \\
\hline M.26 & $39.7 \mathrm{a}$ & 140 & 58.9 a & 94 & $8.42 \mathrm{~b}$ & 102 & $43.6 \mathrm{a}$ & 132 & $37.7 \mathrm{a}$ & 114 \\
\hline P 22 & $22.2 \mathrm{~cd}$ & 78 & $36.6 \mathrm{~b}$ & 58 & $12.0 \mathrm{a}$ & 144 & $20.9 \mathrm{~d}$ & 63 & $22.9 \mathrm{de}$ & 69 \\
\hline P 59 & $14.0 \mathrm{e}$ & 49 & $31.7 \mathrm{bc}$ & 50 & $11.6 \mathrm{a}$ & 140 & $22.2 \mathrm{~d}$ & 67 & 19.9 ef & 60 \\
\hline P 61 & $16.4 \mathrm{de}$ & 58 & $41.3 \mathrm{~b}$ & 66 & $7.43 \mathrm{~b}$ & 90 & $12.3 \mathrm{e}$ & 37 & 19.4 ef & 58 \\
\hline P 62 & $23.9 \mathrm{bc}$ & 85 & $59.5 \mathrm{a}$ & 95 & $12.5 \mathrm{a}$ & 151 & $31.9 \mathrm{~b}$ & 97 & $32.0 \mathrm{ab}$ & 97 \\
\hline P 66 & $30.2 \mathrm{~b}$ & 107 & $38.7 \mathrm{~b}$ & 62 & $7.96 \mathrm{~b}$ & 96 & $32.6 \mathrm{~b}$ & 99 & $27.4 \mathrm{~cd}$ & 83 \\
\hline P 67 & $31.8 \mathrm{~b}$ & 112 & $61.1 \mathrm{a}$ & 97 & $12.1 \mathrm{a}$ & 146 & $39.4 \mathrm{a}$ & 119 & $36.1 \mathrm{a}$ & 109 \\
\hline PB. 4 & $13.4 \mathrm{e}$ & 47 & $22.8 \mathrm{c}$ & 36 & $8.66 \mathrm{~b}$ & 105 & $18.6 \mathrm{de}$ & 56 & $15.9 \mathrm{f}$ & 48 \\
\hline B.396 & $26.6 \mathrm{bc}$ & 94 & $63.1 \mathrm{a}$ & 100 & $7.66 \mathrm{~b}$ & 92 & $31.4 \mathrm{bc}$ & 95 & $32.2 \mathrm{ab}$ & 97 \\
\hline Pure 1 & $25.9 \mathrm{bc}$ & 91 & $54.5 \mathrm{ab}$ & 87 & $12.3 \mathrm{a}$ & 148 & $25.0 \mathrm{~cd}$ & 76 & $29.4 \mathrm{bc}$ & 89 \\
\hline Mean & 24.8 & & 48.3 & & 9.90 & & 28.3 & & 27.8 & \\
\hline
\end{tabular}

Note. ${ }^{1}$ - the yield of trees on M.9 rootstock was considered as $100 \% ;{ }^{2}$ - means followed by the same letter in each column are not significantly different at $P \leq 0.05$ by Duncan's multiple range test.

rootstocks were the most dwarfing in all locations. Low yields in Latvia did not allow the researchers to draw the conclusions on rootstock effect in this country, though this did not influence the average rootstock effects over all locations.

The average cumulative yield efficiency was the highest in Lithuania followed by Estonia, Poland and Latvia (Table 3). Low yields and relatively strong tree growth in Latvia caused very low average efficiency. Rootstock effect on the cumulative yield efficiency was clearly modified by the effect of location. All rootstocks in Poland and almost all rootstocks in Latvia had higher yield efficiency than M.9. More diverse results were recorded in Lithuania and Estonia. Trees on Pure 1 rootstock were the most efficient in Lithuania and Latvia, highly efficient in Poland but not in Estonia. High cumulative yield efficiency of trees on $\mathrm{P} 66$ rootstock was established in all locations except Lithuania. Trees on M.26 rootstock had significantly lowest cumulative yield efficiency in Lithuania, Latvia and Estonia, but not in Poland.

Table 3. Rootstock effect on the cumulative yield $\left(\mathrm{kg} \mathrm{cm}^{-2}\right)$ efficiency, 2010

\begin{tabular}{|c|c|c|c|c|c|c|c|c|c|c|}
\hline Rootstock & Poland & $\begin{array}{c}\text { Relative } \\
\text { efficiency } \\
\%{ }^{1}\end{array}$ & Lithuania & $\begin{array}{c}\text { Relative } \\
\text { efficiency } \\
\%\end{array}$ & Latvia & $\begin{array}{c}\text { Relative } \\
\text { efficiency } \\
\%\end{array}$ & Estonia & $\begin{array}{c}\text { Relative } \\
\text { efficiency } \\
\%\end{array}$ & $\begin{array}{l}\text { Average } \\
\text { over four } \\
\text { locations }\end{array}$ & $\begin{array}{c}\text { Relative } \\
\text { efficiency } \\
\% \\
\end{array}$ \\
\hline M.9 & $1.53 \mathrm{f}^{2}$ & 100 & $2.55 \mathrm{bc}$ & 100 & $0.42 \mathrm{e}$ & 100 & $2.17 \mathrm{bc}$ & 100 & $1.67 \mathrm{c}$ & 100 \\
\hline M.26 & $1.98 \mathrm{~cd}$ & 130 & $1.67 \mathrm{e}$ & 65 & $0.34 \mathrm{e}$ & 81 & $1.60 \mathrm{~d}$ & 74 & $1.40 \mathrm{~d}$ & 84 \\
\hline P 22 & $2.43 \mathrm{ab}$ & 159 & $2.41 \mathrm{~cd}$ & 94 & $0.78 \mathrm{bc}$ & 185 & $2.31 \mathrm{~b}$ & 106 & $1.98 \mathrm{a}$ & 119 \\
\hline P 59 & $2.72 \mathrm{a}$ & 178 & $2.95 \mathrm{ab}$ & 116 & $0.82 \mathrm{~b}$ & 195 & $2.31 \mathrm{~b}$ & 106 & $2.20 \mathrm{a}$ & 132 \\
\hline P 61 & $2.07 \mathrm{~cd}$ & 135 & $2.98 \mathrm{ab}$ & 117 & $0.82 \mathrm{~b}$ & 194 & $2.31 \mathrm{~b}$ & 107 & $2.05 \mathrm{a}$ & 122 \\
\hline P 62 & $2.18 \mathrm{bc}$ & 142 & $2.25 \mathrm{cde}$ & 88 & $0.78 \mathrm{bc}$ & 187 & $1.76 \mathrm{~cd}$ & 81 & $1.74 \mathrm{bc}$ & 104 \\
\hline P 66 & $2.09 \mathrm{~cd}$ & 137 & $1.82 \mathrm{de}$ & 72 & $0.55 \mathrm{~d}$ & 132 & $2.87 \mathrm{a}$ & 132 & $1.83 \mathrm{bc}$ & 110 \\
\hline P 67 & $2.15 \mathrm{bc}$ & 140 & $2.48 \mathrm{bc}$ & 97 & $0.66 \mathrm{~cd}$ & 157 & $2.48 \mathrm{ab}$ & 114 & $1.94 \mathrm{ab}$ & 116 \\
\hline PB. 4 & $2.19 \mathrm{bc}$ & 143 & $2.12 \mathrm{cde}$ & 83 & $1.25 \mathrm{a}$ & 297 & $2.46 \mathrm{ab}$ & 113 & $2.01 \mathrm{a}$ & 120 \\
\hline B.396 & 1.70 ef & 111 & $2.39 \mathrm{~cd}$ & 94 & $0.40 \mathrm{e}$ & 95 & $2.27 \mathrm{~b}$ & 104 & $1.69 \mathrm{c}$ & 101 \\
\hline Pure 1 & $1.99 \mathrm{cde}$ & 130 & $3.14 \mathrm{a}$ & 123 & $0.89 \mathrm{~b}$ & 212 & $1.81 \mathrm{~cd}$ & 83 & $1.96 \mathrm{a}$ & 117 \\
\hline Mean & 2.09 & & 2.43 & & 0.70 & & 2.21 & & 1.86 & \\
\hline
\end{tabular}

Note. ${ }^{1}$ - the cumulative yield efficiency index of trees on M.9 rootstock was considered as $100 \% ;{ }^{2}$ - means followed by the same letter in each column are not significantly different at $P \leq 0.05$ by Duncan's multiple range test. 
Fruit quality. Mean fruit weight differed among locations being the highest in Poland $(239 \mathrm{~g})$ and smallest in Estonia (160 g) (Table 4). In spite of the great differences in average fruit weight between locations, rootstock effect on fruit weight was relatively similar (except Latvia). P 62 has the biggest fruit weight in all locations. The lowest fruit weight was recorded on P 59 except Latvia. Lower fruit weight could be explained by the highest fertility index recorded on P 59. On average, fruit weight on all other rootstocks was not significantly different from M.9.

Table 4. Rootstock effect on mean fruit weight (g) (average of 2006-2010)

\begin{tabular}{ccccccccccc}
\hline Rootstock & Poland & $\begin{array}{c}\text { Relative } \\
\text { weight } \\
\%^{1}\end{array}$ & Lithuania & $\begin{array}{c}\text { Relative } \\
\text { weight \% }\end{array}$ & Latvia & $\begin{array}{c}\text { Relative } \\
\text { weight \% }\end{array}$ & Estonia & $\begin{array}{c}\text { Relative } \\
\text { weight \% } \%\end{array}$ & $\begin{array}{c}\text { Average } \\
\text { over four } \\
\text { locations }\end{array}$ & $\begin{array}{c}\text { Relative } \\
\text { weight \% }\end{array}$ \\
\hline M.9 & $247 \mathrm{ab}^{2}$ & 100 & $211 \mathrm{ab}$ & 100 & $180 \mathrm{ab}$ & 100 & $159 \mathrm{a}$ & 100 & $199 \mathrm{ab}$ & 100 \\
M.26 & $257 \mathrm{ab}$ & 104 & $229 \mathrm{ab}$ & 109 & $158 \mathrm{~b}$ & 88 & $170 \mathrm{a}$ & 107 & $204 \mathrm{ab}$ & 102 \\
P 22 & $217 \mathrm{bc}$ & 88 & $199 \mathrm{ab}$ & 94 & $195 \mathrm{ab}$ & 108 & $161 \mathrm{a}$ & 101 & $193 \mathrm{ab}$ & 97 \\
P 59 & $191 \mathrm{c}$ & 77 & $185 \mathrm{~b}$ & 88 & $199 \mathrm{a}$ & 111 & $151 \mathrm{a}$ & 95 & $182 \mathrm{~b}$ & 91 \\
P 61 & $231 \mathrm{bc}$ & 94 & $206 \mathrm{ab}$ & 98 & $212 \mathrm{a}$ & 118 & $153 \mathrm{a}$ & 96 & $201 \mathrm{ab}$ & 101 \\
P 62 & $268 \mathrm{a}$ & 108 & $225 \mathrm{a}$ & 106 & $212 \mathrm{a}$ & 118 & $177 \mathrm{a}$ & 111 & $221 \mathrm{a}$ & 111 \\
P 66 & $226 \mathrm{abc}$ & 92 & $201 \mathrm{ab}$ & 95 & $180 \mathrm{ab}$ & 100 & $159 \mathrm{a}$ & 100 & $192 \mathrm{ab}$ & 96 \\
P 67 & $235 \mathrm{abc}$ & 95 & $216 \mathrm{ab}$ & 102 & $189 \mathrm{ab}$ & 105 & $167 \mathrm{a}$ & 105 & $202 \mathrm{ab}$ & 101 \\
PB.4 & $246 \mathrm{ab}$ & 100 & $188 \mathrm{ab}$ & 89 & $197 \mathrm{a}$ & 109 & $152 \mathrm{a}$ & 95 & $196 \mathrm{ab}$ & 98 \\
B.396 & $261 \mathrm{ab}$ & 105 & $220 \mathrm{ab}$ & 104 & $197 \mathrm{a}$ & 110 & $154 \mathrm{a}$ & 97 & $208 \mathrm{ab}$ & 105 \\
Pure 1 & $252 \mathrm{ab}$ & 102 & $195 \mathrm{ab}$ & 92 & $192 \mathrm{ab}$ & 107 & $156 \mathrm{a}$ & 98 & $199 \mathrm{ab}$ & 100 \\
Mean & 239 & & 207 & & 192 & & 160 & & 200 & \\
\hline
\end{tabular}

Note. ${ }^{1}$ - the mean fruit weight of trees on M.9 rootstock was considered as $100 \% ;{ }^{2}$ - means followed by the same letter in each column are not significantly different at $P \leq 0.05$ by Duncan's multiple range test.

More intensive fruit colouring was recorded in Estonia (Table 5). Cooler weather in the northernmost trial location and bigger differences between night and day temperatures before harvesting were favourable for increased fruit blush. On average, 60\% of 'Ligol' fruits in Poland and Lithuania had a blush over half of their surface, whereas in Estonia the amount of coloured fruits increased up to $71 \%$. Fruit colouring depended on rootstock genotype, but rootstock effects were different throughout trial locations. If the greatest differences among rootstocks were 24-29\% in Estonia and Poland, results in Lithuania were more contrasting. There, the amount of fruits with the blush more than $50 \%$ was twice as high as in case of PB.4. In spite of the differences between the locations, some rootstocks had steady positive or negative effect on fruit colour in all places. The best fruit colour was achieved from trees on PB.4, whereas less fruit blush was recorded from trees on M.26.

Table 5. Rootstock effect on fruit colour (\%) of fruits with $>50 \%$ blush (average of 2008-2010)

\begin{tabular}{|c|c|c|c|c|c|c|c|c|}
\hline Rootstock & Poland & $\begin{array}{l}\text { Relative } \\
\text { colour } \%{ }^{1}\end{array}$ & Lithuania & $\begin{array}{l}\text { Relative } \\
\text { colour \% }\end{array}$ & Estonia & $\begin{array}{l}\text { Relative } \\
\text { colour \% }\end{array}$ & $\begin{array}{l}\text { Average } \\
\text { over three } \\
\text { locations }\end{array}$ & $\begin{array}{l}\text { Relative } \\
\text { colour \% }\end{array}$ \\
\hline M.9 & $58.2 \mathrm{bc}^{2}$ & 100 & $46.2 \mathrm{de}$ & 100 & $76 \mathrm{~b}$ & 100 & $60.1 \mathrm{bc}$ & 100 \\
\hline M.26 & $48.4 \mathrm{c}$ & 83 & $42.3 \mathrm{de}$ & 92 & $68 \mathrm{bc}$ & 89 & $52.9 \mathrm{c}$ & 88 \\
\hline P 22 & $66.8 \mathrm{ab}$ & 115 & $45.8 \mathrm{de}$ & 99 & $56 \mathrm{c}$ & 74 & $56.2 \mathrm{c}$ & 94 \\
\hline P 59 & $75.1 \mathrm{a}$ & 129 & $79.0 \mathrm{ab}$ & 171 & $66 \mathrm{bc}$ & 87 & $73.4 \mathrm{ab}$ & 122 \\
\hline P 61 & $52.7 \mathrm{c}$ & 91 & $79.3 \mathrm{ab}$ & 172 & $76 \mathrm{~b}$ & 100 & $69.3 \mathrm{~b}$ & 115 \\
\hline P 62 & $66.5 \mathrm{ab}$ & 114 & $37.8 \mathrm{e}$ & 82 & $62 \mathrm{bc}$ & 82 & $55.4 \mathrm{c}$ & 92 \\
\hline P 66 & $55.6 \mathrm{bc}$ & 95 & $66.2 \mathrm{bc}$ & 143 & $72 \mathrm{bc}$ & 95 & $64.6 \mathrm{bc}$ & 107 \\
\hline P 67 & $58.5 \mathrm{bc}$ & 101 & $58.5 \mathrm{~cd}$ & 127 & $80 \mathrm{ab}$ & 105 & $65.7 \mathrm{~b}$ & 109 \\
\hline PB.4 & $65.8 \mathrm{ab}$ & 113 & $92.8 \mathrm{a}$ & 201 & $94 \mathrm{a}$ & 124 & $84.2 \mathrm{a}$ & 140 \\
\hline B.396 & 59.4 bc & 102 & $51.9 \mathrm{de}$ & 112 & $58 \mathrm{c}$ & 76 & $56.4 \mathrm{c}$ & 94 \\
\hline Pure 1 & 58.4 bc & 100 & $54.2 \mathrm{~cd}$ & 117 & $82 \mathrm{ab}$ & 108 & $64.9 \mathrm{bc}$ & 108 \\
\hline Mean & 60.5 & & 59.5 & & 71.8 & & 63.9 & \\
\hline
\end{tabular}

Note. ${ }^{1}$ - the share of fruits with $>50 \%$ blush from trees on M.9 rootstock was considered as $100 \% ;{ }^{2}-$ means followed by the same letter in each column are not significantly different at $P \leq 0.05$ by Duncan's multiple range test.

\section{Discussion}

Usually, growth of trees on M.9 rootstock is standard in most countries in Europe. Sometimes trees on M.9 rootstock exhibit too vigorous growth in places where deep soils prevail (Vercammen, Gomand, 2011). In Northern countries winterhardiness of M.9 is doubtful. All the tested rootstocks except M.26 which was too vigorous demonstrated a desirable growth range: trees on them were similar or weaker than on M.9. Some doubts appear only with super-dwarfing rootstocks PB.4, P 61 and P 59. On average, their TCSA reached only $40-51 \%$ compared to trees on M.9. Only very vigorous apple cultivars should be grafted on these rootstocks, and at the 
same time trees must be planted very densely. But in this case profitability of orchard should be calculated. The performance of super-dwarfing $\mathrm{P}$ series rootstocks was similar to that in the trials conducted earlier in Poland (Bielicki et al., 2007; Zurawicz et al., 2011).

In our previous rootstock trial tree growth reduction was noticed moving from South to North (Kviklys et al., 2012). The same tendency was recorded in the current trial taking into consideration locations in Lithuania, Latvia and Estonia. Average TCSA of trees in Lithuania was by $25 \%$ higher than in Latvia, and TCSA of trees in Latvia was by $15 \%$ higher than in Estonia. Poor tree growth in Poland could be explained by replant problems. Trees were planted in the place previously used for an orchard and it is known that replant conditions usually negatively affect tree growth (Lanauskas et al., 2008). On average, as has been reported in numerous trials, tree efficiency is adversely related to tree growth. Rootstocks that induce lower tree growth determine higher yield efficiency (Hirst et al., 2001; Piestrzeniewicz et al., 2009). Apple trees on the super-dwarfing P 22, P 59, P 61 and PB.4 rootstocks together with dwarfing rootstocks Pure 1 and $P 67$ had significantly higher cumulative yield efficiency on average over all locations. Confirming our results rootstock $\mathrm{P} 67$ was found to be one of the most efficient in several trials performed in Poland too (Zurawicz et al., 2011).

The tendency to smaller fruits moving from South to North was apparently due to a shorter vegetation period with increasing geographical latitude - from Poland, through Lithuania and Latvia, to Estonia. Fruits of cv. 'Ligol' require longer ripening period and higher sum of active temperatures whose sum is lower in Latvia and especially in Estonia. A consistent rootstock effect on mean fruit weight over different locations seems to be arguable in most trials (Marini, 2002; Al-Hinai, Roper, 2004; Jadczuk et al., 2007). Our results of rootstock effect on fruit weight varied among locations, too. However, some data confirm the tendency of smaller fruits from trees on P 59 rootstock (Czynczyk et al., 2009). The negative effect of some other super-dwarfing and highly productive rootstocks such as PB.4 or P 22 on fruit weight was recorded by other researches too (Tomala et al., 2008).

Rootstock effect on fruit coloration was apparently related to crop load, tree vigour and fruit size, which has been recorded in different trials (Barden, Marini, 2001). Rootstock effect on fruit colour was modified by trial location, too. Nevertheless, rootstock BP.4 determined higher percentage of fruits with more intensive blush over all trial locations. Apple trees on super-dwarfing rootstocks PB.4, P 59, P 61 and P 22 had the highest yield efficiency (18-29\% higher than M.9) and a minor decrease in fruit weight. Anyway, considering drastic growth reduction (up to $40-63 \%$ of trees on M.9), only P 22 might be the right decision for high density plantings in very fertile soil conditions, whereas vegetative tree growth should be limited. Rootstock winterhardiness is one of the limiting factors in North East European countries. Such shortcomings of M.9 were recorded in some trials and in commercial orchards therefore more hardy rootstocks are in favour (Wertheim, 1998). Although special hardiness tests of new rootstocks have not been performed, we might expect rootstocks from Polish breeding program or originated in Latvia and Russia to be more winterhardy. Cv. 'Ligol' apple trees on Pure 1 grew weaker than on M.9 and had lower cumulative yield, but higher yield efficiency. Nevertheless, yield efficiency of trees on Pure 1 rootstock was contradictory throughout the trial locations - it was one of the highest in Lithuania and Latvia, and one the lowest in Poland and Estonia.

Growth and productivity parameters of trees on rootstock B.396 were equal to those of trees on M.9. Knowing its higher winterhardiness, rootstock B.396 may replace M.9, which is happening in commercial apple orchards throughout the Baltic countries. Averaged over all trial locations cv. 'Ligol' trees on P 67 rootstock demonstrated moderate growth, the highest cumulative yield (except M.26) and high yield efficiency. Being of the same growth as P 67, rootstock P 66 in all characteristics was behind it. Trees on another rootstock P 62 from the Polish breeding programme exhibited somewhat weaker growth compared with trees on M.9, but had the same cumulative yield and yield efficiency. The tendency of higher mean fruit weight of cv. 'Ligol' apples on P 62 was recorded in all trial locations. The results from the young orchard indicated P 67 to be the most promising rootstock from the new $\mathrm{P}$ series.

\section{Conclusions}

1. According to tree growth control, apple rootstocks are divided into the following groups: superdwarfing - PB.4, P 61, P 59, P 22 and Pure 1, dwarfing M.9, B.396, P 62, P 66 and P 67, semi-dwarfing - M.26.

2. The most vigorous rootstocks (M.26, M.9, P 67, P 62 and B.396) produced the highest cumulative yields in the young orchard.

3 . The tested apple rootstocks had no consistent effect on the mean fruit weight. Significant differences were recorded only between P 62 and P 59 rootstocks.

4. Trial location specific interactions were noticed for all characters tested. Rootstock effect on tree growth, yield and fruit quality is modified by the trial location.

5. Due to the desirable growth vigour, high yield and yield efficiency rootstocks P 67 and B.396 are good replacement of M.9 in the areas where high winterhardiness of rootstocks is required.

\section{Acknowledgements}

Part of this work was carried out within the framework of the long-term research program "Horticulture: agro-biological basics and technologies" implemented by Lithuanian Research Centre for Agriculture and Forestry.

Received 16012013

Accepted 23052013

\section{References}

Al-Hinai Y. K., Roper T. R. 2004. Rootstock effects on growth and quality of 'Gala' apples. HortScience, 39 (6): 12311233

Barden J. A., Marini R. P. 2001. Yield, fruit size, red color, and estimated crop value in the NC-40 1990 cultivar/rootstock 
trial in Virginia. Journal of the American Pomological Society, 55: 154-158

Bielicki P., Czynczyk A., Chlebowska D. 2007. Effect of new polish clonal rootstocks on growth, yield and fruit quality of two apple cultivars. Acta Horticulturae (ISHS), 732: 191-196

Czynczyk A., Jakubowski T. 2007. Value of standard and new selected rootstocks for apples in Poland. Acta Horticulturae (ISHS), 732: 51-57

Czynczyk A., Bielicki P., Bartosiewicz B. 2009. Results of growing three apple cultivars grafted on a number of polish and english rootstocks and their subclones. Journal of Fruit and Ornamental Plant Research, 17 (2): 73-83

Fazio G., Aldwinckle H. S., Robinson T. L., Wan Y. 2011. Implementation of molecular marker technologies in the Apple Rootstock Breeding program in Geneva - challenges and successes. Acta Horticulturae (ISHS), 903: 61-68

Hirst P. M., Autio W. R., Barden J. A., Brown G. R., Crassweller R. M., Domoto P. A., Schupp J. R. 2001. Performance of trees in the $1990 \mathrm{NC}-140$ apple cultivar/rootstock planting: Additional cultivars and rootstocks. Journal of the American Pomological Society, 55: 178-184

Hrotkó K. 2007. Advances and challenges in fruit rootstock research. Acta Horticulturae (ISHS), 732: 33-42

Jadczuk E., Pietranek A., Sadowski A. 2007. Early performance of 'Golden Delicious' apple trees on four rootstocks. Acta Horticulturae (ISHS), 732: 203-206

Kviklys D., Kviklienè N., Bite A., Lepsis J., Univer T., Univer N., Uselis N., Lanauskas J., Buskienė L. 2012. Baltic fruit rootstock studies: evaluation of 12 apple rootstocks in North-East Europe. Horticultural Science (Prague), 39 (1): 1-7
Lanauskas J., Kviklys D., Sakalauskaitė J. 2008. Biological and technological aspects of apple replant disease management in Lithuania. Sodininkystė ir daržininkystè, 27 (3): 135141

Marini R. P. 2002. Does rootstock influence apple fruit size? Compact Fruit Tree, 35 (1): 8-10

Piestrzeniewicz C., Sadowski A., Dziuban R. 2009. Suitability of different dwarfing rootstocks for 'Rubin' apple trees grown in fertile soil. Journal of Fruit and Ornamental Plant Research, 17 (2): 53-62

Sadowski A., Grochowalski W., Słowinski A., Żołtowski J. 2000. Estimation of vigour of apple trees on PB-4 rootstock. Zeszyty Naukowe Instytutu Sadownictwa i Kwiaciarstwa w Skierniewicach, 8: 71-76

Samus V., Gadziev S., Poplavskiy V., Drabudko N. 2006. Rootstocks of fruit crops in Belarus. Sodininkyste ir daržininkystè, 25 (3): 34-38

Tomala K., Andziak J., Jeziorek K., Dziuban R. 2008. Influence of rootstock on the quality of 'Jonagold' apples at harvest and after storage. Journal of Fruit and Ornamental Plant Research, 16: 31-38

Vercammen J., Gomand A. 2011. Search for a more dwarfing rootstock for 'Jonagold' apple. Acta Horticulturae (ISHS), 903: 355-362

Zurawicz E., Bielicki P., Czynczyk A., Bartosiewicz B., Buczek M., Lewandowski M. 2011. Breeding of apple rootstocks in Poland - the latest results. Acta Horticulturae (ISHS), 903: 143-150

Wertheim S. J. 1998. Rootstock guide: apple, pear, cherry, European plum. Fruit Research Station, Wilhelminadorp, The Netherlands

ISSN 1392-3196 / e-ISSN 2335-8947

Zemdirbyste-Agriculture, vol. 100, No. 4 (2013), p. 441-446

DOI 10.13080/z-a.2013.100.056

\title{
Vaismedžių poskiepių tyrimai Baltijos šalyse: naminės obels (Malus domestica Borkh.) naujų poskiepių įvertinimas
}

\author{
D. Kviklys ${ }^{1}$, N. Kviklienè ${ }^{1}$, P. Bielicki ${ }^{2}$, A. Bite ${ }^{3}$, J. Lepsis $^{3}$, T. Univer ${ }^{4}$, N. Univer ${ }^{4}$, \\ N. Uselis ${ }^{1}$, J. Lanauskas ${ }^{1}$ \\ ${ }^{1}$ Lietuvos agrarinių ir miškų mokslų centro Sodininkystès ir daržininkystės institutas \\ ${ }^{2}$ Lenkijos sodininkystès ir daržininkystès institutas \\ ${ }^{3}$ Purès sodininkystès tyrimų centras, Latvija \\ ${ }^{4}$ Estijos gyvybès mokslų universiteto Polli sodininkystès tyrimų centras
}

\section{Santrauka}

Vykdant tarptautinę programą „Vaismedžių poskiepių tyrimai Baltijos šalyse“, 2005-2010 m. Estijoje, Latvijoje, Lietuvoje ir Lenkijoje tirti obelų vegetatyviniai poskiepiai M.26, M.9, P 22, P 59, P 61, P 62, P 66, P 67, B.396, PB.4 bei Pure 1 su 'Ligol' veisle. Vaismedžių, skiepytų i poskiepius PB.4, P 61, P 59, P 22 ir Pure 1, augumas buvo mažesnis nei vaismedžių, augintų su poskiepiu M.9. Vaismedžių, skiepytų i poskiepius P 62, P 66, P 67 ir B.396, augumas buvo toks pats kaip su poskiepiu M.9, o vaismedžiai su poskiepiu M.26 buvo augiausi. Visų tyrimo vietų vidutiniais duomenimis, obelys, augintos su nykštukiniais P 22, P 59, P 61 bei PB.4 ir su žemaūgiais Pure 1 bei P 67 poskiepiais, buvo iš esmès produktyviausios, tačiau poskiepių ịtaka vaismedžių produktyvumui priklausė nuo tyrimo vietos. Poskiepio įtaka vaisiaus vidutinei masei buvo neesminè: vidutiniais duomenimis, didžiausius vaisius užaugino vaismedžiai su poskiepiu P 62, mažiausius - su P 59. Poskiepių įtaka vaisių nusispalvinimui priklausė nuo tyrimo vietos. Atsižvelgiant ị tinkamą vaismedžio augumo ribojimą, didelị derlių bei produktyvumą ir žinant jų ištvermingumą žiemą, šiaurinèse obelų auginimo zonose poskiepiai B.396 ir P 67 gali pakeisti poskiepi M.9.

Reikšminiai žodžiai: augumas, derlius, produktyvumas, vaisių kokybė. 